\title{
Far-infrared detection of methylene ${ }^{\star}$
}

\author{
E. T. Polehampton ${ }^{1}$, K. M. Menten ${ }^{1}$, S. Brünken ${ }^{2}$, G. Winnewisser ${ }^{2}$, and J.-P. Baluteau ${ }^{3}$ \\ 1 Max-Planck-Institut für Radioastronomie, Auf dem Hügel 69, 53121 Bonn, Germany \\ e-mail: epoleham@mpifr-bonn.mpg.de \\ 2 Physikalisches Institut, Universität zu Köln, 50937 Cologne, Germany \\ ${ }^{3}$ Laboratoire d'Astrophysique de Marseille, CNRS \& Université de Provence, BP 8, 13376 Marseille Cedex 12, France
}

Received 5 July 2004 / Accepted 1 October 2004

\begin{abstract}
We present a clear detection of $\mathrm{CH}_{2}$ in absorption towards the molecular cloud complexes Sagittarius B2 and W49 N using the ISO Long Wavelength Spectrometer. These observations represent the first detection of its low excitation rotational lines in the interstellar medium. Towards Sagittarius B2, we detect both ortho and para transitions allowing a determination of the total $\mathrm{CH}_{2}$ column density of $N\left(\mathrm{CH}_{2}\right)=(7.5 \pm 1.1) \times 10^{14} \mathrm{~cm}^{-2}$. We compare this with the related molecule, $\mathrm{CH}$, to determine $\left[\mathrm{CH} / \mathrm{CH}_{2}\right]=2.7 \pm 0.5$. Comparison with chemical models shows that the $\mathrm{CH}$ abundance along the line of sight is consistent with diffuse cloud conditions and that the high $\left[\mathrm{CH} / \mathrm{CH}_{2}\right]$ ratio can be explained by including the effect of grain-surface reactions.
\end{abstract}

Key words. infrared: ISM - ISM: molecules - molecular data - ISM: individual objects: Sagittarius B2 -

ISM: individual objects: W49

\section{Introduction}

Methylene $\left(\mathrm{CH}_{2}\right)$ is thought to be a relatively abundant molecule in diffuse as well as in dense interstellar clouds, with similar abundances to $\mathrm{CH}$ (e.g. van Dishoeck \& Black 1986; Lee et al. 1996). However, it has proved very difficult to detect observationally, mainly due to the inaccessibility of its rotational lines from the ground.

Methylene was initially proposed to explain an unidentified ultraviolet (UV) band observed in comets (Herzberg 1942a,b) but later this band was shown to be associated with $\mathrm{C}_{3}$ (Douglas 1951). $\mathrm{CH}_{2}$ remains undetected in comets, but recently, several of its electronic bands have been observed in the interstellar medium (ISM) by Lyu et al. (2001) using the Hubble Space Telescope. They tentatively detected $\mathrm{CH}_{2}$ absorption bands in the UV spectrum towards two stars, HD154368 and $\zeta$ Oph.

Interstellar searches for rotational emission/absorption are hampered by the peculiar spectrum of $\mathrm{CH}_{2}$, caused by its lightness and $b$-type selection rules (Michael et al. 2003). This results in lines at widely varying wavelengths, all of which are either completely unobservable from the ground or are in difficult spectral regions that are at the edges of atmospheric windows or for which there are few telescopes with suitable instrumentation to observe them.

* Based on observations with ISO, an ESA project with instruments funded by ESA Member States (especially the PI countries: France, Germany, the Netherlands and the UK) with the participation of ISAS and NASA.
Hollis et al. (1995) have, so far, made the only unambiguous identification of $\mathrm{CH}_{2}$ in the ISM, confirming their earlier detection (Hollis et al. 1989). They clearly identified the $4_{04}-3_{13}$ rotational transition with simultaneous measurements of multiple fine-structure features between 68 and $71 \mathrm{GHz}$. These were detected in emission towards Orion KL and W51 M, both dense "hot core" sources, which provide excitation to the $4_{04}$ level which is $\approx 215 \mathrm{~K}$ above the ground state. The lines occur in a rarely observed spectral region, close to the telluric 55-65 $\mathrm{GHz} \mathrm{O}_{2}$ bands. Accurate frequencies for this transition were measured by Lovas et al. (1983).

Low-excitation rotational transitions occur in the farinfrared (FIR) region, accessible by the Infrared Space Observatory (ISO) satellite (and in the future by SOFIA and Herschel), and around $320 \mu \mathrm{m}(940 \mathrm{GHz})$, a spectral region just being opened from the ground. Although limited laboratory data on the rotational spectrum of $\mathrm{CH}_{2}$ have existed for many years (Lovas et al. 1983; Sears et al. 1984), much more accurate frequencies are currently being measured at the Cologne Laboratory for Molecular Spectroscopy (Michael et al. 2003; Brünken et al. 2004).

We have used these new data to search for low-lying rotational transitions of $\mathrm{CH}_{2}$ observed by the ISO Long Wavelength Spectrometer (LWS; Clegg et al. 1996). We clearly detect the strongest fine structure components of transitions from the lowest energy level of both ortho and para $\mathrm{CH}_{2}$ towards Sagittarius B2 (Sgr B2) and of ortho $\mathrm{CH}_{2}$ towards W49 N. Both Sgr B2 and W49 are giant molecular cloud complexes emitting 
strong FIR continuum spectra. This makes them particularly good targets for detecting absorption lines from intervening interstellar matter.

Sgr B2 was observed as part of a wide spectral survey using the LWS Fabry-Pérot (FP) mode, allowing us to search for transitions from all the other low-lying energy levels, as well as to compare the data with absorption from the chemically related $\mathrm{CH}$ molecule. We use the strong $\mathrm{CH}$ lines as a template to fit and calculate column densities for $\mathrm{CH}_{2}$. In Sects. 4 and 5 we present the results towards Sgr B2 and in Sect. 6 detail the results for $\mathrm{W} 49 \mathrm{~N}$ and several other sources where $\mathrm{CH}_{2}$ was not detected. We then compare the results with chemical models in Sect. 7 .

\section{2. $\mathrm{CH}_{2}$ rotational spectrum}

Figure 1 shows the low-lying rotational states of $\mathrm{CH}_{2}$. The energy levels are denoted by $N_{\mathrm{KaKc}}$, where $N$ is the rigid-body rotational quantum number. Each of these levels is split by electron spin-spin and spin-rotation interactions into three finestructure levels, with the quantum number for total angular momentum excluding nuclear spin, $J$, equal to $N+1, N, N-1$ for $N>0(J=1$ at $N=0)$. Due to the presence of two protons with opposite nuclear spin, $\mathrm{CH}_{2}$ has both ortho (nuclear spin quantum number, $I=1)$ and para $(I=0)$ forms. In the ortho levels, the non-zero nuclear spin interacts with the electrons to cause a further hyperfine splitting with total angular momentum quantum number, $F=J+1, J, J-1$. Selection rules for rotational transitions are $\Delta J=0, \pm 1$ and $\Delta F=0, \pm 1$.

Frequencies for low-lying rotational transitions have been calculated from Laser Magnetic Resonance (LMR) laboratory spectra by Sears et al. (1984) with a quoted accuracy of $5 \mathrm{MHz}$ $\left(0.6 \mathrm{~km} \mathrm{~s}^{-1}\right)$. In order to confirm the lines and to calculate accurate line strengths we have used new measurements of the sub-mm lines (Michael et al. 2003; Brünken et al. 2004) to determine more accurate frequency values. Table 1 shows the calculated wavelengths, Einstein coefficients and line strengths (averaging over the hyper-fine structure in ortho transitions). The accuracy of the calculated wavelengths is highest for those transitions actually measured in the lab $\left(2_{11}-2_{02}(153 \mu \mathrm{m})\right.$ and $1_{10}-1_{01}(156 \mu \mathrm{m})$; Brünken et al. 2004). The uncertainty in the calculated Einstein values and line strengths is dominated by that of the dipole moment. We have followed previous authors and used a value of $0.57 \mathrm{D}$ (derived from an ab initio calculation by Bunker \& Langhoff 1983). The calculation strongly depends on the electronic and geometric structure of the molecule and inserting the most recent values for the geometry suggests that the uncertainty must be at least $0.02 \mathrm{D}$ (leading to $\sim 5 \%$ uncertainty in the Einstein coefficients). The line strength in Table 1 is defined in the same way as by Sears et al. (1984), allowing a direct comparison. This shows that the strengths calculated here are half those previously published. However, our results can reproduce the values for the $1_{11}-2_{02}(317 \mu \mathrm{m})$ transition given by Michael et al. (2003) and agree with the calculations of Chandra (1984) (both of these authors used an independent method to calculate their line strengths). This indicates that the line strengths quoted in the paper by Sears et al. (1984)

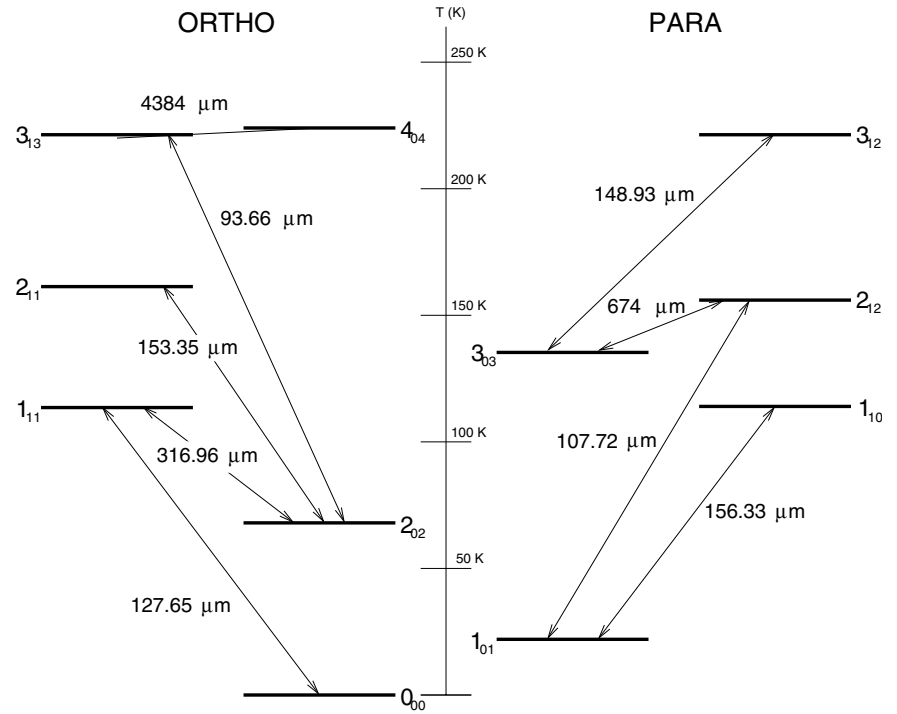

Fig. 1. Low-lying rotational levels of the $\mathrm{CH}_{2}$ molecule showing the wavelength of the strongest fine structure component for each transition.

are likely to be in error by a factor of two (T. Sears, private communication).

\section{Observations and data reduction}

The Sgr B2 observations were carried out as part of a wide spectral survey using the ISO LWS Fabry-Pérot (FP) mode, L03. The whole LWS spectral range, from 47 to $196 \mu \mathrm{m}$ $(6.38-1.53 \mathrm{THz})$, was covered using 36 separate observations with a spectral resolution of $30-40 \mathrm{~km} \mathrm{~s}^{-1}$. The first results from this survey have recently been presented by Ceccarelli et al. (2002); Polehampton et al. (2002); Vastel et al. (2002); Polehampton et al. (2003). We also analysed additional L04/L03 mode observations towards Sgr B2, W49 N, Sgr A* and NGC 7023 and one grating L01 observation towards W49 N, downloaded from the ISO Data Archive ${ }^{1}$. Details of the ISO TDT numbers of all the observations used in this paper are shown in Table A.1.

The LWS beam has an effective diameter of $\sim 80^{\prime \prime}$ (Gry et al. 2003) and was centred on the coordinates shown in Table 2. The coordinates chosen for Sgr B2 were slightly offset from the main FIR peak at Sgr B2 (M) to ensure that Sgr B2 (N) was excluded from the beam. Three positions were observed towards NGC 7023 - the illuminating star (coordinates given in Table 2), the NW PDR $\left(-24.5^{\prime \prime},+39.2^{\prime \prime}\right)$ and the SW PDR $\left(-25.8^{\prime \prime},-63.1^{\prime \prime}\right)$.

The L03 observations towards Sgr B2 were reduced using the ISO Offline pipeline (OLP) version 8 with the remaining observations processed with OLP version 10 (for FP observations there is no significant difference between OLP 8 and 10). Further processing was then applied interactively using routines that appeared as part of the LWS Interactive Analysis package version 10 (LIA10: Lim et al. 2002) and the ISO Spectral Analysis Package (ISAP: Sturm et al. 1998).

${ }^{1}$ see http://www.iso.vilspa.esa.es/ida. 
Table 1. Calculated wavelengths, Einstein coefficients $\left(A_{i j}\right)$ and line strengths $\left(S_{i j}\right)$ for the low-lying transitions of $\mathrm{CH}_{2}$ (ignoring hyperfine-structure in the ortho transitions). The estimated error corresponding to the last significant figure of the calculated wavelengths is given in parenthesis. The Einstein coefficients and line strengths calculated here are half those given by Sears et al. (1984). The detected lines towards Sgr B2 are shown in bold.

\begin{tabular}{|c|c|c|c|}
\hline Transition & $\begin{array}{c}\text { Wavelength } \\
(\mu \mathrm{m})\end{array}$ & $\begin{array}{c}A_{i j} \\
\left(\mathrm{~s}^{-1}\right)\end{array}$ & 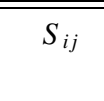 \\
\hline $1_{11}-0_{00} J=0-1$ & $127.31450(6)$ & 0.0163 & 0.989 \\
\hline $1_{11}-0_{00} J=1-1$ & $127.85823(4)$ & 0.0163 & 3.02 \\
\hline $1_{11}-0_{00} J=2-1$ & $127.64614(5)$ & 0.0163 & 4.99 \\
\hline $2_{11}-2_{02} J=1-1$ & $153.102312(6)$ & 0.0105 & 3.31 \\
\hline $2_{11}-2_{02} J=1-2$ & $154.302721(7)$ & 0.00351 & 1.14 \\
\hline $2_{11}-2_{02} J=2-1$ & $152.621311(6)$ & 0.00217 & 1.14 \\
\hline $2_{11}-2_{02} J=2-2$ & $153.814187(6)$ & 0.00962 & 5.15 \\
\hline $2_{11}-2_{02} J=2-3$ & $152.992296(6)$ & 0.00216 & 1.14 \\
\hline $2_{11}-2_{02} J=3-2$ & $154.178941(6)$ & 0.00151 & 1.14 \\
\hline $2_{11}-2_{02} J=3-3$ & $153.352914(7)$ & 0.0125 & 9.25 \\
\hline $3_{13}-2_{02} J=2-1$ & $93.5838(1)$ & 0.0298 & 3.60 \\
\hline $3_{13}-2_{02} J=2-2$ & 94.0309 (1) & 0.00559 & 0.685 \\
\hline $3_{13}-2_{02} J=2-3$ & $93.7231(2)$ & 0.000170 & 0.0205 \\
\hline $3_{13}-2_{02} J=3-2$ & $93.7025(2)$ & 0.0317 & 5.37 \\
\hline $3_{13}-2_{02} J=3-3$ & 93.3967 (2) & 0.00392 & 0.657 \\
\hline $3_{13}-2_{02} J=4-3$ & $93.6621(2)$ & 0.0356 & 7.75 \\
\hline $1_{10}-1_{01} J=0-1$ & $156.43947(1)$ & 0.0133 & 0.500 \\
\hline $1_{10}-1_{01} J=1-0$ & $155.66632(1)$ & 0.00462 & 0.513 \\
\hline $1_{10}-1_{01} J=1-1$ & $157.56539(1)$ & 0.00318 & 0.366 \\
\hline $1_{10}-1_{01} J=1-2$ & $156.76825(1)$ & 0.00548 & 0.622 \\
\hline $1_{10}-1_{01} J=2-1$ & $157.12503(2)$ & 0.00323 & 0.615 \\
\hline $1_{10}-1_{01} J=2-2$ & $156.33233(1)$ & 0.0101 & 1.88 \\
\hline $2_{12}-1_{01} J=1-0$ & $107.2947(2)$ & 0.0134 & 0.487 \\
\hline $2_{12}-1_{01} J=1-1$ & $108.1935(2)$ & 0.0103 & 0.385 \\
\hline $2_{12}-1_{01} J=1-2$ & $107.8170(2)$ & 0.000731 & 0.0270 \\
\hline $2_{12}-1_{01} J=2-1$ & $107.8573(2)$ & 0.0184 & 1.13 \\
\hline $2_{12}-1_{01} J=2-2$ & $107.4832(2)$ & 0.00603 & 0.367 \\
\hline $2_{12}-1_{01} J=3-2$ & $107.7203(2)$ & 0.0244 & 2.10 \\
\hline
\end{tabular}

The interactive processing included the calculation of accurate dark currents (including stray light), careful removal of the LWS grating profile from the spectra and removal of glitches caused by cosmic ray impacts (see Polehampton et al. 2002, 2003). The wavelength scale of each observation was then corrected to the local standard of rest and data co-added for each line (small multiplicative factors were sometimes necessary to match each observation in flux before co-adding). The final uncertainty in wavelength is less than $0.004 \mu \mathrm{m}$ (or $11 \mathrm{~km} \mathrm{~s}^{-1}$ ) corresponding to the error in absolute wavelength calibration (see Gry et al. 2003). Finally, a polynomial baseline was divided into the data to obtain the line-to-continuum ratio. For
Table 2. Coordinates of observed sources.

\begin{tabular}{ccc}
\hline \hline Source & RA $(\mathrm{J} 2000)$ & Dec $(\mathrm{J} 2000)$ \\
\hline Sgr B2 & $17^{\mathrm{h}} 47^{\mathrm{m}} 21.75^{\mathrm{s}}$ & $-28^{\circ} 23^{\prime} 14.1^{\prime \prime}$ \\
W49 N & $19^{\mathrm{h}} 10^{\mathrm{m}} 13.55^{\mathrm{s}}$ & $+09^{\circ} 06^{\prime} 14.7^{\prime \prime}$ \\
Sgr A & $17^{\mathrm{h}} 45^{\mathrm{m}} 39.97^{\mathrm{s}}$ & $-29^{\circ} 00^{\prime} 23.6^{\prime \prime}$ \\
NGC $7023^{\mathrm{h}}$ & $21^{\mathrm{h}} 01^{\mathrm{m}} 36.91^{\mathrm{s}}$ & $+68^{\circ} 09^{\prime} 48.2^{\prime \prime}$ \\
\hline
\end{tabular}

the Sgr B2 data, a 3rd order polynomial was necessary to fit the continuum around and between the detected lines. For the L04 data towards $\mathrm{W} 49 \mathrm{~N}$ where the continuum coverage is much lower, a 1st order baseline was used. The use of low order polynomials and careful masking of the detected lines for the fit ensured that no spurious features were introduced into the data by the division. This step in the reduction is very useful because it effectively bypasses the large uncertainty in absolute flux level caused by multiplicative calibration steps (see Swinyard et al. 1998). The remaining error is dominated by statistical noise in the data with a small additional uncertainty due to the dark current determination and continuum fit.

In Sect. 6, we use one grating L01 observation towards $\mathrm{W} 49 \mathrm{~N}$ to determine the column density of $\mathrm{CH}$. This observation was reduced using OLP 10, and corrected for saturation in the LWS detectors (necessary due to the strength of the W49 N thermal continuum) by T. Grundy at the UK ISO Data Centre. The dataset was further reduced using the LWS L01 postprocessing pipeline which performs several additional corrections detailed in Lloyd et al. (2003). This allowed us to calculate an accurate value for the line-to-continuum ratio in the line.

\section{Sgr B2 results}

We searched the Sgr B2 spectrum for all the low-lying rotational lines of both ortho- and para- $\mathrm{CH}_{2}$ occurring within the survey range $(47-196 \mu \mathrm{m})$. Figure 2 shows the data around the three lowest energy transitions (fits to the line shapes are also shown - these are described in Sect. 4.4). At the spectral resolution of the LWS FP, the fine structure splitting of each rotational transition is resolved but the spacing of the hyperfine components in ortho- $\mathrm{CH}_{2}\left(<10 \mathrm{~km} \mathrm{~s}^{-1}\right)$ is too small to be separated. When analysing ortho transitions we have used wavelengths averaged over the hyperfine structure weighted by $A_{i j}$. The wavelengths used are shown in Table 1.

The survey also shows strong absorption due to $\mathrm{CH}$ (shown in the top panel of Fig. 2). We have used these lines as a basis for fitting the observed $\mathrm{CH}_{2}$ line shapes to fix the relative contribution from features along the line of sight. This was necessary because the signal-to-noise in the $\mathrm{CH}_{2}$ detections is not high enough to allow a full fit accounting for different line of sight components separately. The method assumes that there is a constant $\left[\mathrm{CH} / \mathrm{CH}_{2}\right]$ ratio applicable to all line of sight components. 


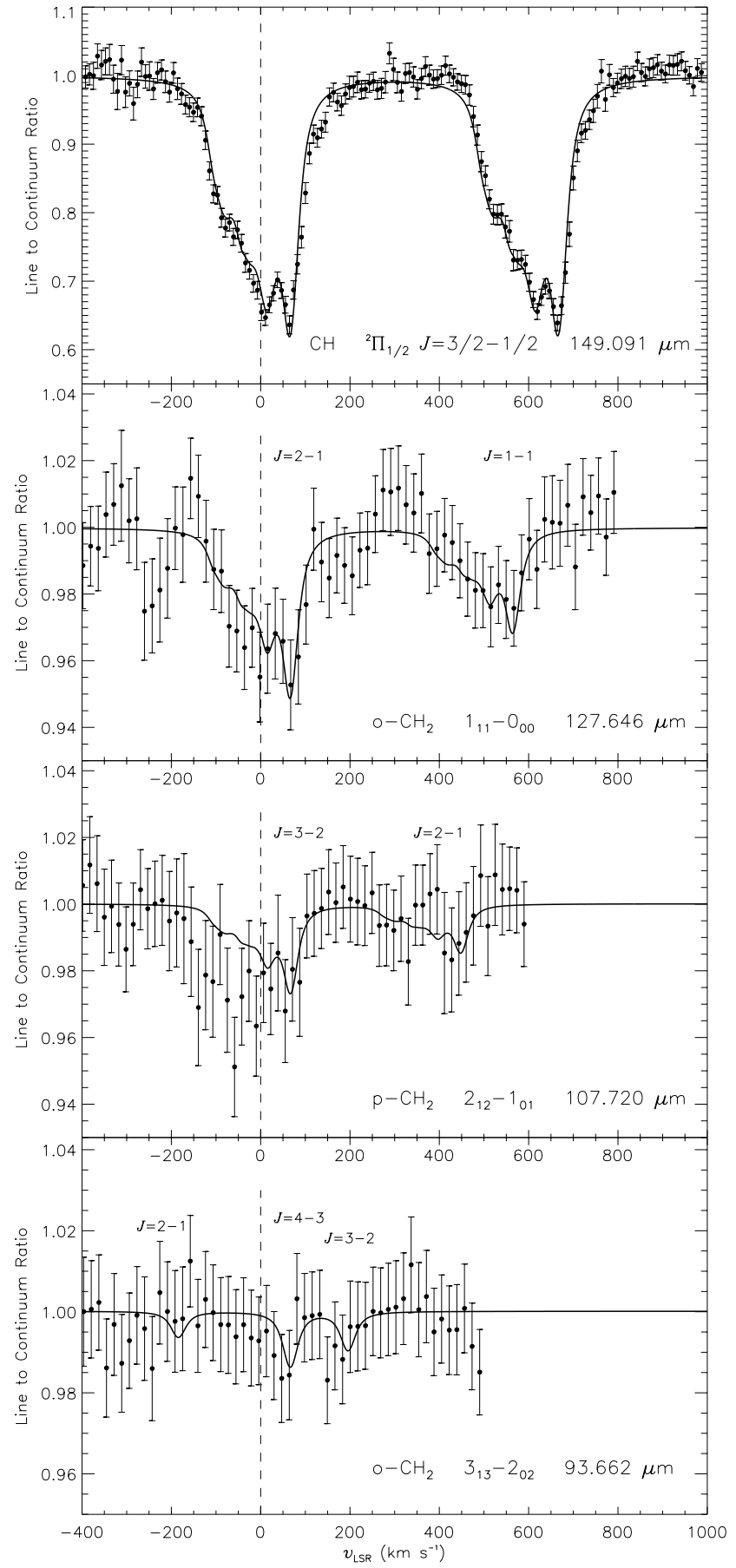

Fig. 2. Upper panel: fit of the $\mathrm{CH}$ line towards Sgr B2 with the data binned at a quarter of the resolution element. Lower panels: in the lower three panels, a model based on the $\mathrm{CH}$ line shape has been fitted to the data around the three lowest energy transitions of $\mathrm{CH}_{2}$. The relative contribution of each velocity component in these fits was fixed to the $\mathrm{CH}$ values in Table 3 , except for the $94 \mu \mathrm{m}\left(3_{13}-2_{02}\right)$ transition in the bottom plot which only includes the velocity component due to Sgr B2 itself (see text). The fit was carried out to the strongest fine structure component in each case. Other (sometimes undetected) components are included in the model using their expected relative line strengths from Table 1. The data are binned at half of the resolution element and the errors reflect both statistical noise and systematic error due to the dark current subtraction and continuum fit. The extra absorption feature seen at $-250 \mathrm{~km} \mathrm{~s}^{-1}$ relative to the $127 \mu \mathrm{m}$ $\left(1_{11}-0_{00}\right)$ transition appears to be real but is not identified.

\section{1. $\mathrm{CH}$}

Two prominent absorption features due to $\mathrm{CH}$ are present in the spectrum due to its ${ }^{2} \Pi_{1 / 2} J=3 / 2-1 / 2$ transition from the ground state to the first rotational level, $\sim 96 \mathrm{~K}$ above ground (see also Cernicharo et al. 1999; Goicoechea et al. 2004). These are due to the $\Lambda$-doublet type splitting of each rotational level and occur at wavelengths of $149.09 \mu \mathrm{m}$ and $149.39 \mu \mathrm{m}$ (Davidson et al. 2001). The two detected lines are shown in the top panel of Fig. 2 and are broad with absorption in the range -150 to $+100 \mathrm{~km} \mathrm{~s}^{-1}$. This is due to galactic spiral arm clouds between the Sun and Galactic Centre (centred at velocities -100 to $+30 \mathrm{~km} \mathrm{~s}^{-1}$, e.g. see Greaves \& Williams 1994) as well as to Sgr B2 itself. The peak absorption occurs near the velocity of Sgr B2 at $\sim 65 \mathrm{~km} \mathrm{~s}^{-1}$. These lines have previously been detected with the Kuiper Airborne Observatory (KAO) by Stacey et al. (1987) but the ISO data provide a significant improvement in the signal-to-noise ratio as well as complete coverage of the neighbouring continuum. No higher transitions of $\mathrm{CH}$ were detected in the survey above the noise.

In order to determine the $\mathrm{CH}$ column density for each velocity component in the line of sight towards Sgr B2, a high resolution model of the line shape was constructed, convolved to the LWS resolution and adjusted to obtain a best fit. This was carried out in the same way as used for $\mathrm{OH}$ lines (see Polehampton et al. 2003,Polehampton et al. in preparation). H I $21 \mathrm{~cm}$ absorption measurements (Garwood \& Dickey 1989) were used to fix the velocity and line width of 10 line of sight components and then optical depths were adjusted in a multiparameter fit to find the minimum in $\chi^{2}$. The optical depth for each component, $\tau$, was calculated from the line-to-continuum ratio using

$I=I_{\mathrm{c}} \exp (-\tau)$

where $I_{\mathrm{c}}$ is the intensity of the continuum. This method assumes that the same velocity components seen in the H I spectrum are present in $\mathrm{CH}$ and $\mathrm{CH}_{2}$ (but the fit does not depend on the H I optical depths derived by Garwood \& Dickey 1989). This is likely because the atomic material is seen to be associated with molecular gas at the same velocities (e.g. Vastel et al. 2002). Each fitted component probably still represents a mean over many narrower features such as those seen in CS absorption with velocity widths $\sim 1 \mathrm{~km} \mathrm{~s}^{-1}$ (Greaves \& Williams 1994). The final fit is shown in the top panel of Fig. 2.

Column densities for each velocity component are shown in Table 3. These were calculated assuming a Doppler line profile with Maxwellian velocity distribution (e.g. Spitzer 1978),

$N_{j}=\frac{8 \pi \sqrt{\pi}}{2 \sqrt{\ln 2}} 10^{17} \frac{\tau_{0} \Delta v}{A_{i j} \lambda_{i j}^{3} g_{i} / g_{j}}$

where $N_{j}$ is the column density in the lower level, $\tau_{0}$ is the optical depth at line centre, $\Delta v$ is the line width in $\mathrm{km} \mathrm{s}^{-1}, A_{i j}$ is the Einstein coefficient for spontaneous emission, $\lambda_{i j}$ is the wavelength in $\mu \mathrm{m}$ and $g_{i}$ is the statistical weight of state $i$.

No higher transitions of $\mathrm{CH}$ have been observed in the spectrum even at the velocity of Sgr B2 itself, showing that the level populations are characterised by a low rotation temperature (i.e. sub-thermal excitation) and the ground state 
Table 3. Results of our fit of the $\mathrm{CH}$ lines towards Sgr B2 are given in column (A). The velocities and line widths are taken from the H I data of Garwood \& Dickey (1989). Column (B) gives the previous estimates of $\mathrm{CH}$ column densities from the KAO measurements of Stacey et al. (1987) and columns (C) and (D) give the results from the radio $\Lambda$-doublet lines from Genzel et al. (1979) and Andrew et al. (1978).

\begin{tabular}{|c|c|c|c|c|c|}
\hline \multirow{2}{*}{$\begin{array}{c}\text { LSR Velocity } \\
\left(\mathrm{km} \mathrm{s}^{-1}\right)\end{array}$} & \multirow{2}{*}{$\begin{array}{c}F W H M \\
\left(\mathrm{~km} \mathrm{~s}^{-1}\right)\end{array}$} & \multicolumn{4}{|c|}{ 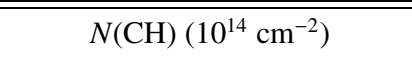 } \\
\hline & & (A) & (B) & (C) & (D) \\
\hline-107.6 & 7 & $0.9 \pm 0.7$ & 4.2 & & \\
\hline-81.7 & 28 & $2.6 \pm 1.1$ & & & 2.5 \\
\hline-51.9 & 17 & $0.3 \pm 0.15$ & & & \\
\hline-44.0 & 8 & $1.5 \pm 0.6$ & 3.6 & & 1.3 \\
\hline-24.4 & 14 & $1.9 \pm 0.4$ & & & 0.3 \\
\hline$+1.1^{a}$ & 19 & $2.0 \pm 0.3$ & 3.2 & & 1.1 \\
\hline+15.7 & 7 & $3.1 \pm 0.8$ & & & 1.5 \\
\hline+31.4 & 21 & $1.5 \pm 1.0$ & & & \\
\hline$+52.8 /+66.7^{b}$ & $11 / 16$ & $9.3 \pm 0.9$ & 5.2 & 3.1 & $9.4^{c}$ \\
\hline Total & & 20.1 & 15.2 & & 16.1 \\
\hline
\end{tabular}

${ }^{a}$ This velocity is corrected from an error in Garwood \& Dickey (1989) where it is given as $+11 \mathrm{~km} \mathrm{~s}^{-1}$.

${ }^{b}$ The H I data resolve 2 components in Sgr B2. However, our fit requires only one of these (at $66.7 \mathrm{~km} \mathrm{~s}^{-1}$ ) to reproduce the ISO spectrum.

${ }^{c}$ This is the combined value for all components $>50 \mathrm{~km} \mathrm{~s}^{-1}$ assuming the emission fills the beam, as described in the text by Andrew et al. (1978).

population is a good measure of the total column density. Using a $2 \sigma$ detection limit for the ${ }^{2} \Pi_{1 / 2} J=5 / 2-3 / 2 \mathrm{CH}$ transition $(116 \mu \mathrm{m})$, we calculate $T_{\text {rot }}<20 \mathrm{~K}$. The fitted optical depths show that the absorption is generally optically thin, although in the Sgr B2 component at $67 \mathrm{~km} \mathrm{~s}^{-1}$ it is marginally optically thick $(\tau=2.4)$. The results of the fit are shown in Fig. 2 and Table 3 , with errors determined by examining $\chi^{2}$ as pairs of optical depths were varied about their best fit values.

One way to check the opacity of Sgr B2 is to use the less abundant ${ }^{13} \mathrm{CH}$ isotopomer whose ${ }^{2} \Pi_{1 / 2} J=3 / 2-1 / 2$ transition has components at $149.79 \mu \mathrm{m}$ and $150.09 \mu \mathrm{m}$ (Davidson et al. 2004). Langer \& Penzias (1990) find a ${ }^{12} \mathrm{C} /{ }^{13} \mathrm{C}$ ratio towards Sgr B2 of $24 \pm 1$ using observations of ${ }^{12} \mathrm{C}^{18} \mathrm{O}$ and ${ }^{13} \mathrm{C}^{18} \mathrm{O}$. However, the ${ }^{13} \mathrm{CH}$ lines are not detected in the ISO spectrum to a limit of $1.3 \%$ of the continuum ( $2 \sigma$ level). This leads to ${ }^{12} \mathrm{CH} /{ }^{13} \mathrm{CH}>36$ at the velocity of Sgr B2. This is higher than the value derived from $\mathrm{CO}$ but this could be due to isotopic fractionation which is expected to increase the ratio from the true ${ }^{12} \mathrm{C} /{ }^{13} \mathrm{C}$ value (see Langer et al. 1984). In the following analysis, we use the column density for Sgr B2 without adjustment.

The final $\mathrm{CH}$ column densities in Table 3 compare well with previous values derived from the KAO observations of same FIR lines by Stacey et al. (1987). Goicoechea et al. (2004) have also examined ISO data of $\mathrm{CH}$ and found absorption extended across the whole surrounding region. They calculate total column densities in the range $(0.8-1.8) \times 10^{15} \mathrm{~cm}^{-2}$, peaking at the central Sgr B2 (M) and Sgr B2 (N) positions. Radio observations of the $\mathrm{CH} \Lambda$-doublet lines by Andrew et al. (1978) are in very good agreement with the individual velocity features that we have fitted - the small discrepancies may be due to their much larger beam size $\left(8.2^{\prime}\right)$, which also included the $(\mathrm{N})$ position. The radio lines were also observed by Genzel et al. (1979), however, they only give the column density for Sgr B2 itself at $+65 \mathrm{~km} \mathrm{~s}^{-1}$ (although they also observed emission from the other velocity components). They derived the column density from the $3264 \mathrm{MHz}$ line giving a value three times lower than ours (however, their weaker data for the $3335 \mathrm{MHz}$ line would give a higher column density).

\subsection{Ortho- $\mathrm{CH}_{2}$}

The lowest ortho level of $\mathrm{CH}_{2}$ occurs at the ground level, $N=0$. We clearly detect absorption from the $1_{11}-0_{00} J=2-1$ and $J=1-1$ fine structure components at $127 \mu \mathrm{m}$ (see Fig. 2). The third component, $J=1-0$, is not detected above the noise in the spectrum. The observed features have a similar shape to the $\mathrm{CH}$ lines, indicating the presence of absorption along the whole line of sight.

The next highest energy level occurs $\sim 67 \mathrm{~K}$ above ground. There are two transitions from this level that occur within the ISO wavelength range at $153 \mu \mathrm{m}\left(2_{11}-2_{02}\right)$ and $94 \mu \mathrm{m}$ $\left(3_{13}-2_{02}\right)$, and one that occurs outside the range at $317 \mu \mathrm{m}$ $\left(1_{11}-2_{02}\right)$. The strongest fine-structure component of these is the $2_{11}-2_{02} J=3-3$ line at $153.353 \mu \mathrm{m}$. However, this wavelength is very close to the $N=2-1 J=3-2$ transition of $\mathrm{NH}$ at $153.344 \mu \mathrm{m}$ (separation $\sim 17 \mathrm{~km} \mathrm{~s}^{-1}$ ) and there is a strong absorption line detected (see Cernicharo et al. 2000). The identification of this feature with $\mathrm{NH}$ is secure because we also detect its $J=2-1$ and $J=1-0$ lines with relative depths that agree extremely well with the predicted line strengths (measured values are 1.0/0.55/0.27 compared to the predicted relative line strengths of 1.0/0.54/0.23 from the JPL line catalogue; Pickett et al. 1998). Furthermore, the next strongest $\mathrm{CH}_{2}$ transition at $153.814 \mu \mathrm{m}$ is not detected. This shows that the major contribution to the $153.35 \mu \mathrm{m}$ line must be from $\mathrm{NH}$ rather than $\mathrm{CH}_{2}$.

The next strongest transition is $31_{13}-2_{02}$ and the $J=4-3$ line $(93.662 \mu \mathrm{m})$ should be almost as strong as the $153.35 \mu \mathrm{m}$ line discussed above. There appear to be some features at roughly the correct velocity at a level $\sim 3 \sigma$ above the statistical noise (see Fig. 2 but note that the error bars shown include systematic uncertainty).

No higher level ortho transitions were detected above the noise in the data.

\subsection{Para- $\mathrm{CH}_{2}$}

The lowest energy state for para- $\mathrm{CH}_{2}$ occurs at $\sim 23 \mathrm{~K}$ above ground (see Fig. 1) and has two transitions at $156 \mu \mathrm{m}\left(1_{01}-1_{10}\right)$ and $107 \mu \mathrm{m}\left(2_{12}-1_{10}\right)$. The strongest fine-structure component of these is the $2_{12}-1_{10} J=3-2$ transition at $107.720 \mu \mathrm{m}$. There is an absorption feature at approximately the correct wavelength for this line, although it appears to be wider than 
expected in its short wavelength wing. The observed absorption depth is also consistent with the data at the position of the next strongest component, $J=2-1$, at $107.857 \mu$ m (see Fig. 2). Using this detection to predict the optical depth of the strongest $1_{01}-1_{10}$ transition at $156.332 \mu \mathrm{m}(J=2-2)$ gives a value consistent with the noise level in the spectrum.

No higher para transitions were detected above the noise in the data.

\section{4. $\mathrm{CH}_{2}$ column densities}

In order to fit the $\mathrm{CH}_{2}$ lines, we assumed that the relative column densities between each velocity feature were the same as for $\mathrm{CH}$. This fixes the shape of the line and allows a single parameter fit to determine an "average" $\mathrm{CH} / \mathrm{CH}_{2}$ column density ratio for each $\mathrm{CH}_{2}$ energy level. The fit was carried out to the strongest fine structure component in each case, with the relative importance of the other (sometimes undetected) fine structure transitions fixed using the line strengths from Table 1. The results of these fits are shown in the lower 3 panels of Fig. 2 (including the undetected transitions, to show that their predicted absorption is consistent with non-detection in the data).

This method worked well for the strongest transition $\left(1_{11}-0_{00}\right)$ at $127 \mu \mathrm{m}$, where both the line shape and relative strength of the $J=2-1$ and $J=1-1$ components show a good fit. A detection limit for the $J=0-1$ component is also consistent with the expected line strengths of 1.0/0.6/0.2 for $J=2-1$, $J=1-1$ and $J=0-1$ respectively. The fact that the line shape agrees well with the data indicates that the $\left[\mathrm{CH} / \mathrm{CH}_{2}\right]$ ratio does not vary significantly between the different line of sight components.

The para transition at $107.7 \mu \mathrm{m}\left(2_{12}-1_{01}\right)$ does not show such good agreement in line shape with the model. As mentioned in Sect. 4.3, the main line appears wider than expected, although the noise is high in this part of the spectrum. It is unlikely that this extra absorption is associated with the $\mathrm{CH}_{2}$ line as this would mean that the negative velocity features would have to be stronger than the component due to Sgr B2 itself and this is not observed in other lines. It could be due to overlap with an unidentified feature (although we have not been able to find a likely candidate) or an instrumental effect (e.g. the overlap of mini-scans can sometimes cause problems for wide lines - see Polehampton et al. 2003). A final possibility is that the extra absorption could have been introduced when dividing by the polynomial fit to the continuum. However, we minimised this effect by using a low order polynomial (3rd order) to carefully fit only the continuum around features that already appeared in the raw data. Any variation in the fitted baseline is on a larger scale than the excess absorption in the line. In fitting the line, we have only considered velocities above $0 \mathrm{~km} \mathrm{~s}^{-1}$. The actual line shape is not well constrained by these data and our fitting method of fixing the relative contribution of each velocity to that of $\mathrm{CH}$ may overestimate the absorption due to the line of sight clouds.

In order to fit the data around the transition at $94 \mu \mathrm{m}$ $\left(3_{13}-2_{02}\right)$, we modified the line template to include only the velocity component at $+67 \mathrm{~km} \mathrm{~s}^{-1}$ from Table 3 . This is because we only expect a significant population in the higher levels at the velocity of Sgr B2 itself. In Sgr B2 itself, excitation to the $2_{02}$ level ( $\sim 67 \mathrm{~K}$ above ground) can be provided by FIR photons from the strong dust continuum, whereas in the line of sight clouds there is no strong radiation field and the densities are too low for collisional excitation to be important. The bottom panel in Fig. 2 shows the results of the fit.

The small discrepancy in velocity between fit and data can be explained by the fact that the main feature is only $3 \sigma$ above the noise. The uncertainty in the exact line wavelength is also relatively high for this transition as it has never been measured in the lab either in Cologne or by Sears et al. (1984). The difference between the value quoted in Table 1 and that by Sears et al. (1984) is $\sim 3 \mathrm{~km} \mathrm{~s}^{-1}$.

The final column densities for the lowest ortho and para levels of $\mathrm{CH}_{2}$ were determined directly from the fitted $\mathrm{CH} / \mathrm{CH}_{2}$ ratio for each level, assuming that the distribution over fine structure levels is determined by the line strengths given in Table 1. The final best fit values summed over all velocities are,

$N\left(0_{00}\right)=(2.9 \pm 0.3) \times 10^{14} \mathrm{~cm}^{-2}$
$N\left(1_{01}\right)=(3.4 \pm 0.9) \times 10^{14} \mathrm{~cm}^{-2}$.

The column density in the $2_{02}$ level at the velocity of Sgr B 2 is, $N\left(2_{02}\right)_{\mathrm{sgrB} 2}=(1.2 \pm 0.5) \times 10^{14} \mathrm{~cm}^{-2}$. Treating this as an upper limit allows us to make a comparison with the ortho ground state (using only the Sgr B2 component) and estimate the excitation temperature for Sgr B2, giving $T_{\text {rot }}<40_{-11}^{+14} \mathrm{~K}$. In order for absorption to be seen, this excitation temperature must be lower than the temperature of the dust producing the FIR background. In Sgr B2 the dust temperature has been found to be $\sim 30 \mathrm{~K}$ (Goicoechea \& Cernicharo 2001), and so our estimated excitation temperature is consistent within its errors. At this temperature we do not expect any higher levels to be significantly populated and do not detect any of the other higher transitions that occur within the spectral survey range $\left(2_{20}-1_{11}\right.$ at $50.5 \mu \mathrm{m} ; 2_{21}-1_{10}$ at $50.8 \mu \mathrm{m}$ and $3_{12}-3_{03}$ at $\left.148.8 .5 \mu \mathrm{m}\right)$.

Assuming that only the first three energy levels in Sgr B2 are populated, the ortho-to-para ratio in the $+67 \mathrm{~km} \mathrm{~s}^{-1}$ component is $1.6_{-0.6}^{+0.9}$. The equilibrium value of the ratio for $\mathrm{CH}_{2}$ should be $\geq 3$ for low temperatures. This difference could be a relic of the formation process with the current ortho-to-para ratio fixed at formation and/or set by the ratio of the parent species. Alternatively, it could indicate that not all of the $22_{12}$ $1_{01}$ absorption seen at $107.7 \mu \mathrm{m}$ is associated with $\mathrm{CH}_{2}$, possibly due to blending with another line. Reducing the para column density by approximately a factor of 2 would lead to an ortho-to-para ratio of 3.

Overall, the column density summed over all velocities in both ortho and para states is $N\left(\mathrm{CH}_{2}\right)=(7.5 \pm 1.1) \times 10^{14} \mathrm{~cm}^{-2}$, giving a final ratio with $\mathrm{CH}$ of $\left[\mathrm{CH} / \mathrm{CH}_{2}\right]=2.7 \pm 0.5$. If the para column density is reduced to make the ortho-to-para ratio equal to 3 , the $\left[\mathrm{CH} / \mathrm{CH}_{2}\right]$ ratio would be increased to 3.7 .

\section{Abundances}

To discuss $\mathrm{CH}$ and $\mathrm{CH}_{2}$ abundances, we need an estimate of the hydrogen column density towards Sgr B2. 
In the Galactic spiral arm clouds along the line of sight $\left(-100\right.$ to $\left.+30 \mathrm{~km} \mathrm{~s}^{-1}\right), \mathrm{HCN}$ and CS measurements suggest average densities of $200 \mathrm{~cm}^{-3}$, but also the presence of gas up to $10^{4} \mathrm{~cm}^{-3}$ (Greaves 1995). Their structure is probably similar to photodissociation regions (PDRs) with a molecular core and atomic skin at the surface, and UV illumination provided by the mean interstellar radiation field (Vastel et al. 2002). $N\left(\mathrm{H}_{2}\right)$ has been estimated to be $5,9,4$ and $14 \times 10^{21} \mathrm{~cm}^{-2}$ respectively in the $-100,-40,-25$ and $0 \mathrm{~km} \mathrm{~s}^{-1}$ features (Greaves \& Nyman 1996), with corresponding atomic hydrogen column densities approximately equal to 2, 4, 2 and $8 \times 10^{21} \mathrm{~cm}^{-2}$ (Vastel et al. 2002). In order to compare with diffuse cloud models, we calculate the abundances taking into account the total hydrogen particle column, $N_{\mathrm{H}}=N(\mathrm{H})+2 \mathrm{~N}\left(\mathrm{H}_{2}\right)$, although it is difficult to determine whether the observed $\mathrm{CH}$ and $\mathrm{CH}_{2}$ co-exists with both atomic and molecular hydrogen regions - they are probably confined to one or more specific layers within each cloud. Using our fitted column densities for $\mathrm{CH}$ in Table 3, we calculate an approximate abundance of $N(\mathrm{CH}) / N_{\mathrm{H}} \sim(0.6-3) \times 10^{-8}$ for these clouds. According to our average $\left[\mathrm{CH} / \mathrm{CH}_{2}\right]$ ratio, $N\left(\mathrm{CH}_{2}\right) / N_{\mathrm{H}}$ is thus $(0.2-1.1) \times 10^{-8}$.

The situation is more complicated at the velocity of Sgr B2 as there may be contributions to the absorption from layers with widely different conditions. Comito et al. (2003) have found that a significant fraction of the water absorption towards Sgr B2 must be due to a hot (500-700 K), low density layer (also observed in ammonia lines; Ceccarelli et al. 2002; Hüttemeister et al. 1995) but that there must also be absorption from the warm (40-80 K) envelope. Goicoechea $\&$ Cernicharo (2002) have derived a large $\mathrm{OH}$ abundance at the velocity of Sgr B2 suggesting that there is a strong UV field producing clumpy PDRs and a temperature gradient from 40-600 K through the envelope. The total $\mathrm{H}_{2}$ column density in front of the FIR continuum has been estimated from the FIR spectrum to be $(2-10) \times 10^{23} \mathrm{~cm}^{-2}$ (Goicoechea \& Cernicharo 2001). Using this to calculate approximate abundances gives $N(\mathrm{CH}) / N_{\mathrm{H}} \sim(0.5-2) \times 10^{-9}$ and $N\left(\mathrm{CH}_{2}\right) / N_{\mathrm{H}} \sim$ $(0.2-0.7) \times 10^{-9}$.

\section{6. $\mathrm{CH}_{2}$ in other sources}

We have also searched the ISO Data Archive for observations towards other sources that may show the low-lying $\mathrm{CH}_{2}$ lines.

\section{1. $W 49 N$}

Observations that cover the wavelengths of the $\mathrm{CH}_{2}$ lines were carried out using the LWS L04 mode towards the active star forming region, $\mathrm{W} 49 \mathrm{~N}$. This is the strongest IR peak in the W49 A molecular cloud complex which is located at $11.4 \mathrm{kpc}$ from the Sun and $8.1 \mathrm{kpc}$ from the Galactic Centre (Gwinn et al. 1992). Its spectrum shows a strong thermal continuum in the FIR with a peak near $60 \mu \mathrm{m}$ (Vastel et al. 2001). Line emission associated with the molecular cloud itself is centred at $8 \mathrm{~km} \mathrm{~s}^{-1}$ (e.g. Jaffe et al. 1987), but there are also several features due to intervening gas at velocities between 16 and $75 \mathrm{~km} \mathrm{~s}^{-1}$ (e.g. Nyman 1983). Vastel et al. (2000) have mapped the $\mathrm{CO}$ emission in the ISO LWS beam and found 7 velocity

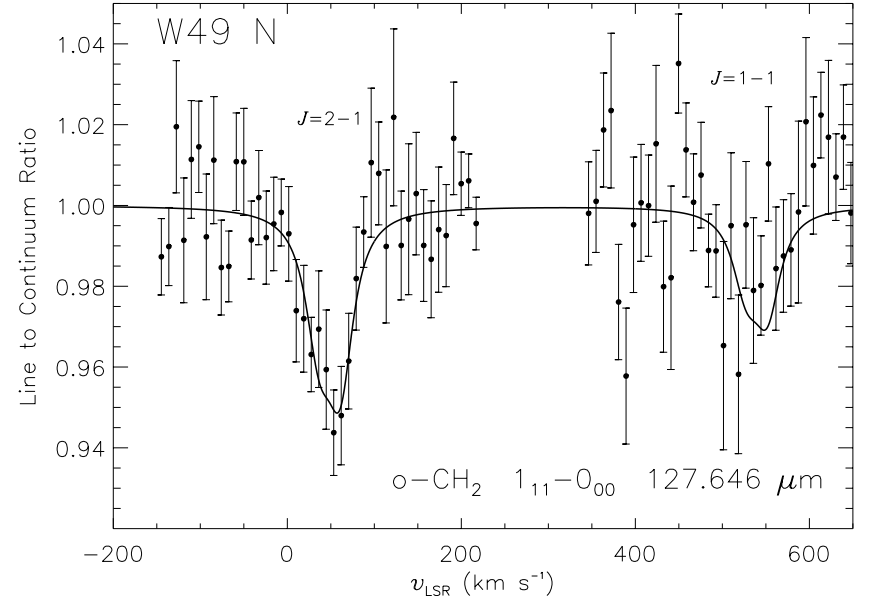

Fig. 3. Data around the $J=2-1(127.6 \mu \mathrm{m})$ and $J=1-1(127.8 \mu \mathrm{m})$ fine structure components of the $1_{11}-0_{00} \mathrm{CH}_{2}$ transition toward $\mathrm{W} 49 \mathrm{~N}$. The solid line shows a model derived from a fit to the $J=2-$ 1 component and the expected relative line strength of the $J=1-1$ component.

components in the range $35-70 \mathrm{~km} \mathrm{~s}^{-1}$. Recently, Plume et al. (2004) have measured $\mathrm{H}_{2} \mathrm{O}$ towards $\mathrm{W} 49$ and the same features show up in absorption. They arise because the line of sight intersects the Sagittarius spiral arm in two places (e.g. Greaves \& Williams 1994).

The strongest ortho- $\mathrm{CH}_{2} 1_{11}-0_{00}$ fine structure component at $127.6 \mu \mathrm{m}(J=2-1)$ is clearly detected in absorption in the LWS FP spectrum. However, the spectral resolution and signal-to-noise ratio in the data do not allow a detailed fit using all the velocity components observed in $\mathrm{CO}$ and $\mathrm{H}_{2} \mathrm{O}$ lines. Therefore, we performed a simplified fit using two components to represent the strongest features seen in the $\mathrm{H}_{2} \mathrm{O}$ spectrum of Plume et al. (2004). We fixed the velocities (and FWHM) to be $37 \mathrm{~km} \mathrm{~s}^{-1}\left(10 \mathrm{~km} \mathrm{~s}^{-1}\right)$ and $61 \mathrm{~km} \mathrm{~s}^{-1}\left(7 \mathrm{~km} \mathrm{~s}^{-1}\right)$ and found the best fitting optical depths after convolving to the LWS resolution. Lower velocity components were not necessary to reproduce the line shape, showing that all the absorption comes from the line of sight clouds. Only the $J=2-1$ component was used for the fit. The result is shown in Fig. 3, which also shows a prediction for the $J=1-1(127.858 \mu \mathrm{m})$ fine structure component based its expected relative line strength. Column densities for the $0_{00}$ level, calculated from the best fitting optical depths and equation 2 , are $(0.8 \pm 0.4) \times 10^{14} \mathrm{~cm}^{-2}$ and $(1.2 \pm 0.4) \times 10^{14} \mathrm{~cm}^{-2}$ at $37 \mathrm{~km} \mathrm{~s}^{-1}$ and $61 \mathrm{~km} \mathrm{~s}^{-1}$ respectively.

The para transition at $107 \mu \mathrm{m}\left(2_{12}-1_{01}\right)$ is not detected above the noise. Assuming that only the two lowest levels are populated and using a $2 \sigma$ limit on the line depth, gives an orthoto-para ratio $>2.1$.

The CH line at $149 \mu$ m was not observed toward W49 N using the FP mode of the LWS, but it was measured with the lower resolution grating mode. At this resolution the $\Lambda$-doublet line splitting, as well as the line of sight structure, is not resolved and we can only estimate a total $\mathrm{CH}$ column density that may include absorption associated with W49 itself, giving $8.4 \times 10^{14} \mathrm{~cm}^{-2}$. Sume \& Irvine (1977) have observed $\mathrm{CH}$ radio $\Lambda$-doublet lines towards W49 $\mathrm{A}$ and they find column densities of $8 \times 10^{13} \mathrm{~cm}^{-2}$ and $10 \times 10^{13} \mathrm{~cm}^{-2}$ in the 


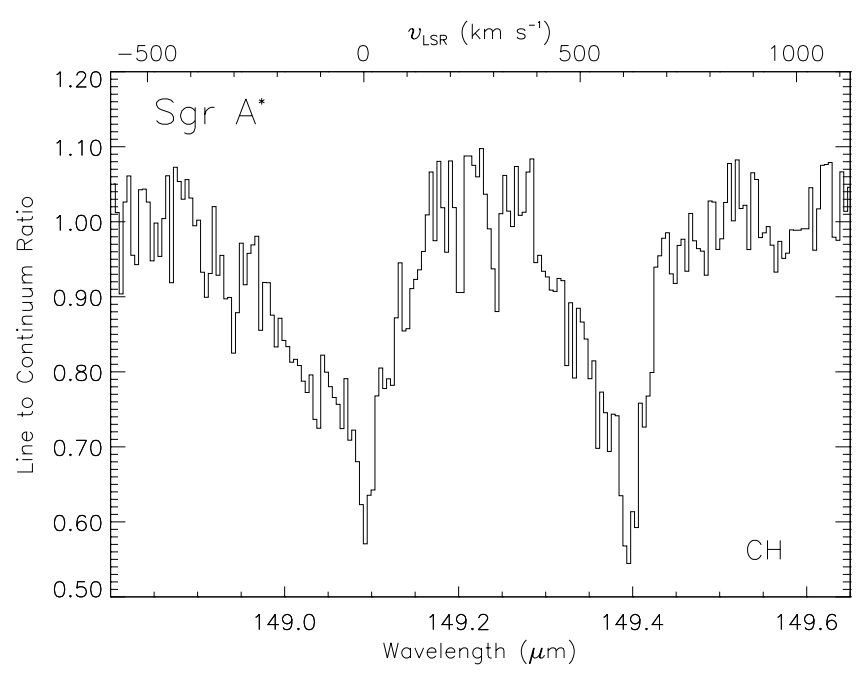

Fig. 4. The $\mathrm{CH}^{2} \Pi_{1 / 2} J=3 / 2-1 / 2$ transition observed towards Sgr $\mathrm{A}^{*}$ showing two resolved $\Lambda$-doublet components.

velocity intervals $30-45 \mathrm{~km} \mathrm{~s}^{-1}$ and $54-72 \mathrm{~km} \mathrm{~s}^{-1}$. Rydbeck et al. (1976) also observed the $\Lambda$-doublet lines, giving column densities of $7 \times 10^{13} \mathrm{~cm}^{-2}$ and $18 \times 10^{13} \mathrm{~cm}^{-2}$. Both authors show that these two velocity intervals give the most important $\mathrm{CH}$ contributions. This means the extra column density seen in the LWS grating measurement probably also has highest contribution from these ranges and the radio measurements may be an underestimate (probably due to the much larger beam size of $15^{\prime}$ used for these observations).

Using the average of the two radio $\mathrm{CH}$ measurements and combining the ortho column density and para upper limit for $\mathrm{CH}_{2}$, gives a lower limit for the $\left[\mathrm{CH} / \mathrm{CH}_{2}\right]$ ratio of 1.4 and 0.8 in the two velocity intervals.

\section{2. $\operatorname{Sgr} A^{*}$}

Observations using the LWS FP mode were also made towards Sgr $\mathrm{A}^{*}$ at the Galactic Centre. It has a FIR continuum showing strong thermal emission from dust, peaking at $\sim 50 \mu \mathrm{m}$. Observations of several hydrocarbons have been reported towards this source, including $\mathrm{CH}_{3}$ (Feuchtgruber et al. 2000). We have searched LWS FP observations for the lines of $\mathrm{CH}_{2}$ and $\mathrm{CH}$ and Fig. 4 shows the observed absorption due to the ${ }^{2} \Pi_{1 / 2} J=3 / 2-1 / 2(149 \mu \mathrm{m})$ rotational transition of $\mathrm{CH}$. These lines show peak absorption near $0 \mathrm{~km} \mathrm{~s}^{-1}$, probably due to local gas. There is also absorption at negative velocities due to galactic spiral arm clouds. This picture is consistent with observations of $\mathrm{CH} \Lambda$-doublet lines towards Sgr A, which showed that the strong Galactic Centre component normally observed in other molecules at $+50 \mathrm{~km} \mathrm{~s}^{-1}$ is relatively weak in $\mathrm{CH}$ (Genzel et al. 1979). At the wavelength of the ortho $\mathrm{CH}_{2} 1_{11}-0_{00}$ transition $(127 \mu \mathrm{m})$, the signal-to-noise ratio in the data is rather low compared to the Sgr B2 observations and we do not detect any absorption. The para $2_{12}-1_{01}$ transition at $107.7 \mu \mathrm{m}$ is also not detected. Combining $2 \sigma$ upper limits for both transitions, we calculate a lower limit to the $\left[\mathrm{CH} / \mathrm{CH}_{2}\right]$ ratio at $0 \mathrm{~km} \mathrm{~s}^{-1}$ of 2.6.

\subsection{NGC 7023}

The ISO database also contains LWS FP observations of $\mathrm{CH}_{2}$ towards three positions in the NGC 7023 PDR. However, no lines are detected above the noise in the spectra. This is consistent with LWS grating observations in which no $\mathrm{OH}, \mathrm{CH}$ or $\mathrm{CH}_{2}$ were detected toward any position in NGC 7023 (Fuente et al. 2000).

\section{Discussion}

\subsection{Comparison with models}

In Table 4 we summarise the results from several chemical models for diffuse (van Dishoeck \& Black 1986) and dense clouds (Lee et al. 1996), as well as a recent model that takes both gas-phase and grain-surface chemistry into account (Ruffle \& Herbst 2001). In these models, the abundances of $\mathrm{CH}$ and $\mathrm{CH}_{2}$ are generally predicted to be higher towards lower densities and temperatures. We also include the results from a PDR model of the IC63 nebula from Jansen et al. (1995) - this is thought to have similar physical conditions to the Sgr B2 envelope $\left(n \sim 10^{5} \mathrm{~cm}^{-3} ; 650-900\right.$ times the average interstellar UV field). In this model, $\mathrm{CH}$ and $\mathrm{CH}_{2}$ peak towards the edge of the PDR at $A_{\mathrm{v}}<2$, with $\left[\mathrm{CH} / \mathrm{CH}_{2}\right]$ generally $\sim 2$ (although in a model with higher fractional abundance of carbon they calculated a value $\sim 20$ ). The $\mathrm{CH}$ abundance reached is relatively high compared to the dense cloud models with similar $n_{\mathrm{H}}$. In PDR models with higher density and more intense UV fields, even larger $\mathrm{CH}$ and $\mathrm{CH}_{2}$ abundances can be reached in the outer layers (Sternberg \& Dalgarno 1995).

Table 4 compares our estimated abundances with the models. In the line of sight clouds towards Sgr B2 (-100 to $+30 \mathrm{~km} \mathrm{~s}^{-1}$ ), Greaves \& Nyman (1996) have examined the abundances of 11 species observed at $3 \mathrm{~mm}\left(\mathrm{HCO}^{+}, \mathrm{HCN}\right.$, $\mathrm{HNC}, \mathrm{CN}, \mathrm{CCH}, \mathrm{C}_{3} \mathrm{H}_{2}, \mathrm{CS}, \mathrm{SiO}, \mathrm{N}_{2} \mathrm{H}^{+}, \mathrm{CH}_{3} \mathrm{OH}$ and $\mathrm{SO}$ ) and found that overall, the chemistry in these features is similar to the dark cloud TMC1. However, our abundances are much closer to diffuse cloud values, showing that $\mathrm{CH}$ and $\mathrm{CH}_{2}$ may reside in the less dense parts of the clouds. In Sgr B2 itself, our derived abundances are much more uncertain due to the difficulty in estimating $N(\mathrm{H})$, but are lower than the line of sight clouds, consistent with denser material in Sgr B2.

We can also compare our results with the previous observations of $\mathrm{CH}_{2}$ in diffuse (Lyu et al. 2001) and dense clouds (Hollis et al. 1995). Using the results of Lyu et al. (2001) with hydrogen abundances for their lines of sight (see van Dishoeck \& Black 1986; Snow et al. 1996), we calculate value consistent with our line of sight values and the diffuse cloud model listed in Table 4. For the high densities and temperatures prevailing in the Orion-KL and W51 M hot cores, Hollis et al. (1995) 
Table 4. Comparison of model predictions for diffuse and dense clouds with the observed abundances (compared to $2 N\left(\mathrm{H}_{2}\right)+\mathrm{N}(\mathrm{H})$ ) of $\mathrm{CH}$ and $\mathrm{CH}_{2}\left(X(\mathrm{CH})\right.$ and $\left.X\left(\mathrm{CH}_{2}\right)\right)$. The diffuse cloud model is for the line of sight to $\zeta$ Per (van Dishoeck \& Black 1986): vDB86. The quoted density and temperature are for the centre of the cloud in the model. The dense cloud models are steady state values corresponding to the gas phase "new standard model" of Lee et al. (1996): L96 and the gas-grain model of Ruffle \& Herbst (2001): RH01 including photochemistry (P1/A at $10^{8} \mathrm{yr}$ ). The PDR model is for a density of $10^{5} \mathrm{~cm}^{-3}$ and radiation field $\sim 650-900$ times the average interstellar value (Jansen et al. 1995 ): J95. The observational results derived from our ISO measurements are shown as well as previous results from Lyu et al. (2001) and Hollis et al. (1995).

\begin{tabular}{|c|c|c|c|c|c|c|}
\hline & & $\begin{array}{l}\text { Density } \\
\left(\mathrm{cm}^{-3}\right)\end{array}$ & $\begin{array}{c}\text { Temperature } \\
(\mathrm{K})\end{array}$ & $\begin{array}{l}X(\mathrm{CH}) \\
\left(10^{-8}\right)\end{array}$ & $\begin{array}{c}X\left(\mathrm{CH}_{2}\right) \\
\left(10^{-8}\right)\end{array}$ & {$\left[\mathrm{CH} / \mathrm{CH}_{2}\right]$} \\
\hline Model & Diffuse (vDB86) & 500 & 25 & 1.6 & 2.4 & 0.67 \\
\hline \multirow[t]{4}{*}{ predictions: } & Dense (L96) & $10^{3}$ & 10 & 0.25 & 1.6 & 0.018 \\
\hline & Dense (L96) & $10^{5}$ & 10 & 0.0007 & 0.039 & 0.16 \\
\hline & Dense (RH01) & $2 \times 10^{4}$ & 10 & 0.1 & 1.2 & 0.08 \\
\hline & PDR (J95) & $10^{5}$ & & 1.4 & 0.7 & 2.0 \\
\hline \multirow{5}{*}{$\begin{array}{l}\text { Results from } \\
\text { this work: }\end{array}$} & Sgr B2 Line of Sight & & & $0.6-3$ & $0.2-1.1$ & $2.7 \pm 0.5$ \\
\hline & Sgr B2 & & & $0.05-0.2$ & $0.02-0.07$ & $2.7 \pm 0.5$ \\
\hline & W49 N $37 \mathrm{~km} \mathrm{~s}^{-1}$ & & & & & $>1.4$ \\
\hline & $\mathrm{W} 49 \mathrm{~N} 61 \mathrm{~km} \mathrm{~s}^{-1}$ & & & & & $>0.8$ \\
\hline & Sgr $A^{*}$ & & & & & $>2.6$ \\
\hline \multirow{4}{*}{$\begin{array}{l}\text { Previous } \\
\text { observational } \\
\text { results: }\end{array}$} & $\zeta \mathrm{Oph}$ & & & $1.8^{b}$ & $4.3^{a}$ & $0.4^{b}$ \\
\hline & HD154368 & & & $1.9^{d}$ & $0.79^{c}$ & $2.4^{d}$ \\
\hline & Orion-KL & $\sim 10^{5}$ & $100-200$ & $0.06^{e}$ & $0.4^{e}$ & $0.1^{e}$ \\
\hline & W51 M & $\sim 10^{4}$ & $100-200$ & $0.007^{e}$ & $0.07^{e}$ & $0.1^{e}$ \\
\hline
\end{tabular}

${ }^{a}$ Combining $N\left(\mathrm{CH}_{2}\right)$ from Lyu et al. (2001) with a hydrogen column density as quoted in van Dishoeck \& Black (1986).

${ }^{b}$ Based on $N(\mathrm{CH})=2.5 \times 10^{13} \mathrm{~cm}^{-2}$ from van Dishoeck \& Black (1989).

${ }^{c}$ Combining $N\left(\mathrm{CH}_{2}\right)$ from Lyu et al. (2001) with a hydrogen column density from Snow et al. (1996).

${ }^{d}$ Based on $N(\mathrm{CH})=8 \times 10^{13} \mathrm{~cm}^{-2}$ from van Dishoeck \& Black (1989).

${ }^{e}$ Taking values as quoted by Hollis et al. (1995) - but note that $X(\mathrm{CH})$ could be significantly higher - see text.

derive $X\left(\mathrm{CH}_{2}\right) \approx 4 \times 10^{-9}$ and $7 \times 10^{-10}$, respectively, carefully considering beam-filling factors. These values are closer to our results for Sgr B2.

\section{2. $\mathrm{CH} / \mathrm{CH}_{2}$ chemistry}

Both $\mathrm{CH}$ and $\mathrm{CH}_{2}$ are formed by the dissociative recombination of $\mathrm{CH}_{3}^{+}$and primarily destroyed by reaction with atomic oxygen to form HCO, a constituent of many more complex molecules. Hollis et al. (1995) compared their derived $\mathrm{CH}_{2}$ abundance in the Orion-KL and W51 M hot cores with radio observations of $\mathrm{CH}$ to determine branching ratios for the formation of $\mathrm{CH}$ and $\mathrm{CH}_{2}$ of 0.1 and 0.9 respectively. The actual branching ratios for the dissociative recombination of $\mathrm{CH}_{3}^{+}$ have since been measured in the lab by Vejby-Christensen et al. (1997). There are 4 important pathways, with $\mathrm{CH}_{2}$ as the major product (40\%), two reactions producing $\mathrm{CH}(30 \%)$ and the remainder producing atomic carbon $(30 \%)$. This appears to agree with models of diffuse and translucent clouds, which required a high abundance of $\mathrm{CH}_{2}$ to reproduce the measured column densities of $\mathrm{C}_{2}$ (van Dishoeck \& Black 1986, 1989)

The above discussion indicates that $\mathrm{CH}_{2}$ should be more abundant than $\mathrm{CH}$. However, our observations show that in the line of sight towards $\mathrm{Sgr} \mathrm{B} 2, \mathrm{CH}_{2}$ has less than half the abundance of $\mathrm{CH}$ - if this were not the case, we would have detected much stronger $\mathrm{CH}_{2}$ absorption. The observations towards W49 and the non-detection towards Sgr A* also appear to confirm this. Furthermore, the results of Lyu et al. (2001) towards HD154368 combined with previous measurements of $\mathrm{CH}$ (see Table 4) show very good agreement with the Sgr B2 ratio.

These results are contrary to the previous observations towards Orion-KL and W51 M where $\mathrm{CH}_{2}$ was found to be 10 times more abundant than $\mathrm{CH}$. This may be due to the difficulty in determining the $\mathrm{CH}$ column density equivalent to the measured $\mathrm{CH}_{2}$ emission in these sources. Hollis et al. (1995) used CH column densities derived by Rydbeck et al. (1976), giving $N(\mathrm{CH})=2 \times 10^{13} \mathrm{~cm}^{-2}$ towards W51 M. In contrast, Turner (1988) made a detailed study of the $\Lambda$-doublet satellite lines from the first rotationally excited state towards W51, giving a value for the $57 \mathrm{~km} \mathrm{~s}^{-1}$ velocity component that is more than 100 times larger: $6.2 \times 10^{15} \mathrm{~cm}^{-2}$. This indicates that $\left[\mathrm{CH} / \mathrm{CH}_{2}\right]$ could be as high as 30 , although the radio $\mathrm{CH}$ line observations are likely to sample a much larger and cooler region than the $70 \mathrm{GHz} \mathrm{CH}_{2}$ lines which require high temperatures to be excited. 
This shows it is extremely important to have consistent measurements of $\mathrm{CH}$ and $\mathrm{CH}_{2}$ (with transitions at similar energies, using the same beam size and with consistent calibration) in order to be able to make a good comparison. So far the current data towards Sgr B2 provides the best comparison of the two species.

In order to explain the low abundance of $\mathrm{CH}_{2}$ with respect to $\mathrm{CH}$ observed towards $\mathrm{Sgr} \mathrm{B} 2$, we require other formation/destruction processes to be important for the $\mathrm{CH} / \mathrm{CH}_{2}$ balance. The PDR model of Jansen et al. (1995) shows that the presence of strong UV radiation can reproduce our observed $\left[\mathrm{CH} / \mathrm{CH}_{2}\right]$ ratio. This may explain the ratio in Sgr B2 but in the galactic spiral arm clouds, the UV field is much weaker and densities lower than used by Jansen et al. (1995). Viti et al. (2000) have modelled the hydrocarbon chemistry in diffuse and translucent clouds $\left(n=300 \mathrm{~cm}^{-3}\right)$ including the interaction of gaseous $\mathrm{C}^{+}$with dust grains. This leads to the production of hydrocarbons by surface reactions. In some of their models (for $A_{\mathrm{v}}=4$ ), they find column densities approaching those that we find in the line of sight clouds towards Sgr B2 and $\left[\mathrm{CH} / \mathrm{CH}_{2}\right]$ ratios 7.7-13.6. These surface reactions could explain our high observed $\left[\mathrm{CH} / \mathrm{CH}_{2}\right]$ ratio.

\section{Summary}

We have made the first detection of the low-lying rotational transitions of the $\mathrm{CH}_{2}$ molecule in the ISM towards Sgr B2 and W49 $\mathrm{N}$ in absorption. We do not detect $\mathrm{CH}_{2}$ towards $\mathrm{Sgr} \mathrm{A}^{*}$ or NGC 7023. For Sgr B2 we were able to compare these observations with measurements of the ground state rotational lines of $\mathrm{CH}$, providing a good estimate of the total column density of both species along the line of sight. These observations provide the best comparison of the two species to date, giving a $\left[\mathrm{CH} / \mathrm{CH}_{2}\right]$ ratio of $2.7 \pm 0.5$, probably fairly constant in all the observed velocity components. The results towards $\mathrm{W} 49 \mathrm{~N}$ appear to agree with this ratio, giving a lower limit of $\sim 1.0$.

Comparison with chemical models shows that the abundances in the line of sight clouds are close to diffuse cloud conditions whereas in Sgr B2 itself they indicate denser gas is present. Our high $\left[\mathrm{CH} / \mathrm{CH}_{2}\right]$ ratio can be explained by models including grain surface reactions (e.g. Viti et al. 2000).

Future observations in the FIR with SOFIA and in the sub-millimetre with telescopes such as the Atacama Pathfinder Experiment (APEX) will be highly interesting to extend the study of $\mathrm{CH}_{2}$ to other sources.

Acknowledgements. We wish to thank T. W. Grundy (RAL) for supplying us with the LWS grating spectrum of W49 N processed using the latest version of the strong source correction and L01 post-processing pipeline. The LWS Interactive Analysis (LIA) package is a joint development of the ISO-LWS Instrument Team at the Rutherford Appleton Laboratory (RAL, UK - the PI Institute) and the Infrared Processing and Analysis Center (IPAC/Caltech, USA). The ISO Spectral Analysis Package (ISAP) is a joint development by the LWS and SWS Instrument Teams and Data Centres. Contributing institutes are CESR, IAS, IPAC, MPE, RAL and SRON.

\section{Appendix A: Observations used}

Table A.1. Log of the observations used.

\begin{tabular}{|c|c|c|c|c|}
\hline Transition & $\begin{array}{c}\text { Observing } \\
\text { Mode }\end{array}$ & $\begin{array}{l}\text { ISO TDT } \\
\text { Number }\end{array}$ & $\begin{array}{c}\text { LWS } \\
\text { Detector }\end{array}$ & $\begin{array}{c}\text { Resolution } \\
\left(\mathrm{km} \mathrm{s}^{-1}\right)\end{array}$ \\
\hline \multicolumn{5}{|c|}{${ }^{12} \mathrm{CH}$ and ${ }^{13} \mathrm{CH}$ Sgr B2 } \\
\hline$J=3 / 2-1 / 2(149 \mu \mathrm{m})$ & L03 & 50700208 & LW3 & 36 \\
\hline \multirow[t]{4}{*}{$\left(\&\right.$ para- $\left.-\mathrm{CH}_{2} 3_{12}-3_{03}{ }^{a}\right)$} & L03 & 84500102 & LW3 & 36 \\
\hline & L03 & 50600603 & LW3 & 36 \\
\hline & L03 & 50600814 & LW4 & 36 \\
\hline & L03 & 50900521 & LW4 & 36 \\
\hline$J=3 / 2-5 / 2(115 \mu \mathrm{m})^{a}$ & L03 & 50601013 & LW2 & 34 \\
\hline \multicolumn{5}{|l|}{ ortho- $\mathrm{CH}_{2}$ Sgr B2 } \\
\hline \multirow[t]{3}{*}{$1_{11}-0_{00}(127 \mu \mathrm{m})$} & L03 & 50700511 & LW2 & 34 \\
\hline & L03 & 50800819 & LW3 & 34 \\
\hline & L04 & 47600907 & LW2 & 34 \\
\hline \multirow[t]{5}{*}{$2_{11}-2_{02}(153 \mu \mathrm{m})^{a}$} & L03 & 50700208 & LW4 & 35 \\
\hline & L03 & 50800317 & LW4 & 35 \\
\hline & L03 & 50400823 & LW4 & 35 \\
\hline & L03 & 50600405 & LW3 & 35 \\
\hline & L03 & 83600317 & LW3 & 35 \\
\hline \multirow[t]{3}{*}{$3_{13}-2_{02}(94 \mu \mathrm{m})$} & L03 & 50800218 & LW1 & 34 \\
\hline & L03 & 50400823 & LW1 & 34 \\
\hline & L03 & 50700610 & LW1 & 34 \\
\hline $2_{20}-1_{11}(50 \mu \mathrm{m})^{a}$ & L03 & 50800218 & SW2 & 64 \\
\hline \multicolumn{5}{|l|}{ para-CH 2 Sgr B2 } \\
\hline \multirow[t]{3}{*}{$1_{10}-1_{01}(156 \mu \mathrm{m})^{b}$} & L03 & 50700707 & LW4 & 35 \\
\hline & L03 & 50700610 & LW4 & 35 \\
\hline & L03 & 50800317 & LW4 & 35 \\
\hline \multirow[t]{2}{*}{$2_{12}-1_{01}(107 \mu \mathrm{m})$} & L03 & 50800515 & LW2 & 32 \\
\hline & L03 & 50800819 & LW2 & 32 \\
\hline \multirow[t]{4}{*}{$2_{21}-1_{10}(51 \mu \mathrm{m})^{a}$} & L03 & 50800216 & SW2 & 64 \\
\hline & L03 & 50600814 & SW2 & 64 \\
\hline & L03 & 50700511 & SW2 & 64 \\
\hline & L03 & 50900521 & SW2 & 64 \\
\hline \multicolumn{5}{|l|}{$\overline{\mathrm{CH}_{2} \mathrm{~W} 49 \mathrm{~N}}$} \\
\hline $1_{11}-0_{00}(127 \mu \mathrm{m})$ & L04 & 49900406 & LW2 & 34 \\
\hline $2_{12}-1_{01}(107 \mu \mathrm{m})^{a}$ & L04 & 49900406 & LW2 & 32 \\
\hline \multicolumn{5}{|l|}{ CH W49 N } \\
\hline$J=3 / 2-1 / 2(149 \mu \mathrm{m})$ & L01 & 52700702 & LW4 & 1200 \\
\hline \multicolumn{5}{|l|}{$\mathrm{CH}_{2}$ Sgr A $^{*}$} \\
\hline $1_{11}-0_{00}(127 \mu \mathrm{m})^{a}$ & L03 & 50900204 & LW2 & 34 \\
\hline \multirow[t]{4}{*}{$2_{12}-1_{01}(107 \mu \mathrm{m})^{a}$} & L03 & 50800902 & LW2 & 32 \\
\hline & L03 & 85000139 & LW2 & 32 \\
\hline & L03 & 50900444 & LW1 & 32 \\
\hline & L03 & 85000247 & LW1 & 32 \\
\hline \multicolumn{5}{|l|}{ CH Sgr $A^{*}$} \\
\hline$J=3 / 2-1 / 2(149 \mu \mathrm{m})$ & L03 & 84900448 & LW3 & 34 \\
\hline \multicolumn{5}{|c|}{$\overline{C \mathrm{CH}_{2} \text { NGC } 7023 \text { (3 different positions) }}$} \\
\hline \multirow[t]{3}{*}{$1_{11}-0_{00}(127 \mu \mathrm{m})^{a}$} & L04 & 33901603 & LW2 & 34 \\
\hline & L04 & 34601501 & LW2 & 34 \\
\hline & L04 & 56000208 & LW2 & 34 \\
\hline
\end{tabular}

\footnotetext{
${ }^{a}$ Not detected.

${ }^{b}$ Blended with NH line.
} 


\section{References}

Andrew, B. H., Avery, L. W., \& Broten, N. W. 1978, A\&A, 66, 437

Brünken, S., Michael, E. A., Lewen, F., et al. 2004, Can. J. Chem., 82, 676

Bunker, P. R., \& Langhoff, S. R. 1983, J. Molec. Spectrosc., 102, 204

Ceccarelli, C., Baluteau, J.-P., Walmsley, M., et al. 2002, A\&A, 383, 603

Cernicharo, J., Goicoechea, J. R., \& Caux, E. 2000, ApJ, 534, L199

Cernicharo, J., Orlandi, M. A., González-Alfonso, E., \& Leeks, S. J. 1999, in The Universe as seen by ISO, ESA SP-427, 655

Chandra, S. 1984, Ap\&SS, 98, 269

Clegg, P. E., Ade, P. A. R., Armand, C., et al. 1996, A\&A, 315, L38

Comito, C., Schilke, P., Gerin, M., et al. 2003, A\&A, 402, 635

Davidson, S. A., Evenson, K. M., \& Brown, J. M. 2001, ApJ, 546, 330

Davidson, S. A., Evenson, K. M., \& Brown, J. M. 2004, J. Mol. Spectrosc., 223, 20

Douglas, A. E. 1951, ApJ, 114, 466

Feuchtgruber, H., Helmich, F. P., van Dishoeck, E. F., \& Wright, C. M. 2000, ApJ, 535, L111

Fuente, A., Martín-Pintado, J., Roderíguez-Fernández, N. J., Cernicharo, J., \& Gerin, M. 2000, A\&A, 354, 1053

Garwood, R. W., \& Dickey, J. M. 1989, ApJ, 338, 841

Genzel, R., Downes, D., Pauls, T., Wilson, T. L., \& Bieging, J. 1979, A\&A, 73, 253

Goicoechea, J. R., \& Cernicharo, J. 2001, ApJ, 554, L213

Goicoechea, J. R., \& Cernicharo, J. 2002, ApJ, 576, L77

Goicoechea, J. R., Roderíguez-Fernández, N. J., \& Cernicharo, J. 2004, ApJ, 600, 214

Greaves, J. S. 1995, MNRAS, 273, 918

Greaves, J. S., \& Nyman, L. 1996, A\&A, 305, 950

Greaves, J. S., \& Williams, P. G. 1994, A\&A, 290, 259

Gry, C., Swinyard, B., Harwood, A., et al. 2003, ISO Handbook Volume III (LWS), Version 2.1, ESA SAI-99-077/Dc

Gwinn, C. R., Moran, J. M., \& Reid, M. J. 1992, ApJ, 393, 149

Herzberg, G. 1942a, Rev. Mod. Phys., 14, 195

Herzberg, G. 1942b, ApJ, 96, 314

Hollis, J. M., Jewell, P. R., \& Lovas, F. J. 1989, ApJ, 346, 794

Hollis, J. M., Jewell, P. R., \& Lovas, F. J. 1995, ApJ, 438, 259

Hüttemeister, S., Wilson, T. L., Mauersberger, R., et al. 1995, A\&A, 294, 667

Jaffe, D. T., Harris, A. I., \& Genzel, R. 1987, ApJ, 316, 231

Jansen, D. J., van Dishoeck, E. F., Black, J. H., Spaans, M., \& Sosin, C. 1995, A\&A, 302, 223

Langer, W. D., Graedel, T. E., Frerking, M. A., \& Armentrout, P. B. 1984, ApJ, 277, 581
Langer, W. D., \& Penzias, A. A. 1990, ApJ, 357, 477

Lee, H.-H., Bettens, R. P. A., \& Herbst, E. 1996, A\&AS, 119, 111

Lim, T. L., Hutchinson, G., Sidher, S. D., et al. 2002, SPIE, 4847, 435

Lloyd, C., Lerate, M. R., \& Grundy, T. W. 2003, The LWS L01

Pipeline, version 1, available from the ISO Data Archive at http://www.iso.vilspa.esa.es/ida/

Lovas, F. J., Suenram, R. D., \& Evenson, K. M. 1983, ApJ, 267, L131

Lyu, C.-H., Smith, A. M., \& Bruhweiler, F. C. 2001, ApJ, 560, 865

Michael, E. A., Lewen, F., Winnewisser, G., et al. 2003, ApJ, 596, 1356

Nyman, L.-Å. 1983, A\&A, 120, 307

Pickett, H. M., Poynter, R. L., Cohen, E. A., et al. 1998, J. Quant. Spectrosc. \& Rad. Transfer, 60, 883

Plume, R., Kaufman, M. J., Neufeld, D. A., et al. 2004, ApJ, 605, 247

Polehampton, E. T., Baluteau, J.-P., Ceccarelli, C., Swinyard, B. M., \& Caux, E. 2002, A\&A, 388, L44

Polehampton, E. T., Brown, J. M., Swinyard, B. M., \& Baluteau, J.-P. 2003, A\&A, 406, L47

Ruffle, D. P., \& Herbst, E. 2001, MNRAS, 322, 770

Rydbeck, O. E. A., Kollberg, E., Hjalmarson, Å., et al. 1976, ApJS, 31,333

Sears, T. J., McKellar, A. R. W., Bunker, P. R., Evenson, K. M., \& Brown, J. M. 1984, ApJ, 276, 399

Snow, T. P., Black, J. H., van Dishoeck, E. F., et al. 1996, ApJ, 465, 245

Spitzer, L. 1978, Physics of the Interstellar Medium (New York: John Wiley and Sons)

Stacey, G. J., Lugten, J. B., \& Genzel, R. 1987, ApJ, 313, 859

Sternberg, A., \& Dalgarno, A. 1995, ApJS, 99, 565

Sturm, E., Bauer, O. H., Lutz, D., et al. 1998, in Astronomical Data Analysis Software and Systems VII, ASP Conf. Ser., 145, 161

Sume, A., \& Irvine, W. M. 1977, A\&A, 60, 337

Swinyard, B. M., Burgdorf, M. J., Clegg, P. E., et al. 1998, SPIE, 3354, 888

Turner, B. E. 1988, ApJ, 329, 425

van Dishoeck, E. F., \& Black, J. H. 1986, ApJS, 62, 109

van Dishoeck, E. F., \& Black, J. H. 1989, ApJ, 340, 273

Vastel, C., Caux, E., Ceccarelli, C., et al. 2000, A\&A, 357, 994

Vastel, C., Polehampton, E. T., Baluteau, J.-P., et al. 2002, ApJ, 581, 315

Vastel, C., Spaans, M., Ceccarelli, C., Tielens, A. G. G. M., \& Caux, E. 2001, A\&A, 376

Vejby-Christensen, L., Andersen, L. H., Heber, O., et al. 1997, ApJ, 483,531

Viti, S., Williams, D. A., \& O’Neill, P. T. 2000, A\&A, 354, 1062 